\title{
Scan-Driven Fully-Automated Pipeline for Personalized, 3D Printed Low-Cost Prosthetic Hand
}

\author{
Yair Herbst ${ }^{1}$, Shunit Polinsky ${ }^{1}$, Anath Fischer ${ }^{1}$, Yoav Medan ${ }^{2}$, Ronit Schneor ${ }^{1}$, Joshua Kahn ${ }^{1}$, and \\ Alon Wolf ${ }^{1}$, Senior Member, IEEE
}

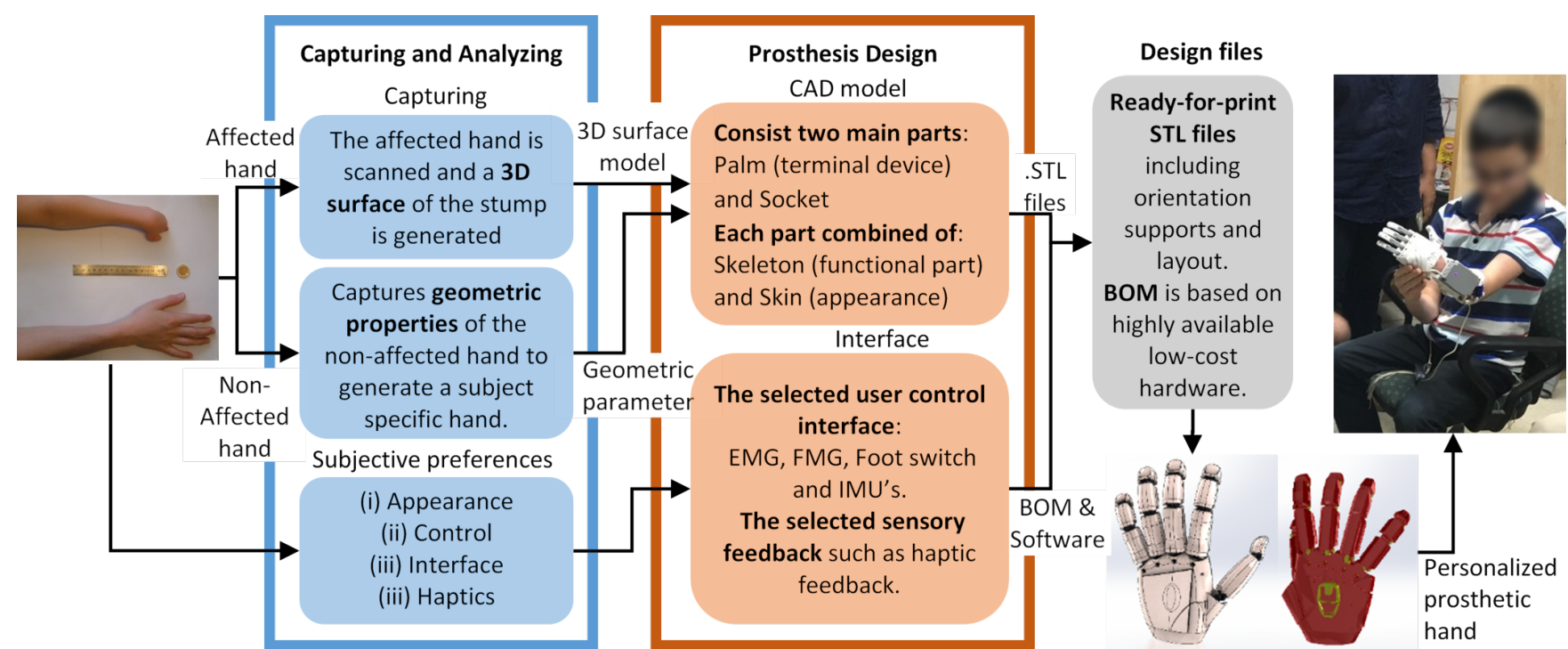

Figure 1. Our proposed low-cost and user-specific hand-fitting pipeline. The pipeline starts with user data which initiates a fully-automated process that ends with a personalized prosthetic hand fitted to the user.

\begin{abstract}
The process of fitting a prosthetic hand that is a comfortable, functional, easy to use, has an acceptable appearance and overall improves the amputees' quality of life is a complex, tedious and costly process. The very high price tag due to the time spent on manually fitting the device by a trained specialist makes these devices inaccessible to large portions of the population. We present a concept and preliminary results for a fully automated fitting and manufacturing pipeline for a personalized low-cost prosthetic hand. The hand is personalized in almost every aspect, from appearance to user interface, control and feedback. The pipeline only requires a 3D printer, a RealSense camera, a few basic mechanical components and basic tools for the model assembly. The user scan-driven data and the user preferences initiate a fully-automated pipeline which culminates in a customized, easy-to-assemble PCB design and ready to print STL files, including the optimized orientation, support and layout, such that the final parts are only one click away. We believe that the proposed pipeline and design can substantially improve quality of life for amputees and could potentially be expanded to other medical applications.
\end{abstract}

\section{INTRODUCTION}

The loss of one's hand can lead to a drastic reduction in the quality of life by decreasing the level of independence

The first and second authors contributed equally to this work.

${ }^{1}$ Authors are with the Mechanical Engineering Department, Technion Israel Institute of Technology, Haifa 3200003, Israel. (e-mail: \{yair.herbst, shunitp, joshua.kahn\}@campus.technion.ac.il, \{meranath, schneor, alonw (a)technion.ac.il)

${ }^{2}$ Yoav Medan is with the Electrical Engineering Department, Technion Israel Institute of Technology, Haifa 3200003, Israel and with Haifa3D organization, Haifa 3303333, Israel. (e-mail: yoav@medan-il.com) and the capability of performing activities of daily living (ADLs) [1]. The cost of a prosthetic hand ranges from $\$ 3,000$ for a body powered prosthesis, to $\$ 100,000$ for a neuro-prosthetic arm such as the i-Limb and the DEKA arm [2]. The very high price tag makes these devices inaccessible to large portions of the population. In many cases, the price is mainly influenced by the time spent on manually fitting the device by a trained specialist. Even when the financial barriers are surpassed, rejection rates of prosthetic devices are considerably high and are usually related to the following causes: lack of social acceptance, weight, sensitivity of the electrical system, lack of stable grip and adaptive grasping force, lack of sensory feedback and age of first fitting (financial resources play a crucial role in prescription of prostheses for children due to constant growth) [1], [3]. Not using a prosthetic device could lead to degeneration of joints and muscles, inflammations and other complications [4]. According to Katsavelis et al. [5] there is a crucial need to develop a functional, easy to fit and maintain and customizable, yet low cost prosthetic.

In recent years, alongside the growing availability of 3D printers and improvements in Computer Aided Design (CAD) programs, medical applications for 3D printing are expanding rapidly and are expected to revolutionize health care in the near future [5], [6]. Using 3D printers for manufacturing prosthetic devices has many advantages. First, a design can be constructed out of fewer parts or even one, reducing costly assembly procedures. Second, 3D printers are capable of creating highly complex geometries, thus providing large design freedom. Unlike traditional prostheses manufacturing techniques, designs can be easily personalized 
and customized [7]. That said, it is important to note the limitations of $3 \mathrm{D}$ printing. It is difficult to predict the mechanical properties of the printed product and even then, there is only a limited amount of materials-for-printing compared to conventional manufacturing materials. One of the most commonly known research and development groups when discussing 3D printed prosthetic hands is the e-NABLE community [8]. The designs created by the community are open-source and the fabrication is done locally using consumer $3 \mathrm{D}$ printers. From the modeling point-of-view, the usage of digital fitting exists in several medical domains such as design and fabrication of maxillofacial implants [9] and custom made hip prostheses using CT scans [10] . Even for lower limb amputation, a software for design and testing of a personalized socket and prosthesis in a fully virtual environment, was developed by Colombo et al. [11].

In the case of prosthetic hands, as in many other consumer products, the design of the product has two different facets - the design of the model itself and the design of user interfaces. In other words, the design of the device includes not only the mechanical and functional design but also the user control methods for producing an intuitive and user-friendly device. Most of the advanced prosthetic hands available rely on electromyogram (EMG) signals from the user's stump [12]. As the devices become more sophisticated and users have more Degrees of Freedoms (DOF) to control, utilizing a control strategy that will not cause cognitive fatigue and lack of reliability is becoming more challenging. The developers of the DEKA Arm, which is characterized by a high number of DOF, conducted research comparing a foot control and EMG pattern recognition control. Their findings suggested that for transradial amputees foot operated motion sensor control (i.e. IMUs) is a more effective control for the DEKA Arm than the EMG control method [13].

In this paper, we present a novel, digital design process to create a personalized and low-cost prosthetic hand. Our proposed fitting pipeline is entirely digital to minimize the design time, the high cost and dependency on trained professionals throughout the pipeline, while potentially achieving a low-cost, tailor-made design that can be accessible from anywhere on the globe. The pipeline is divided into three main steps. The first is obtaining user data using a combination of regular and depth cameras, and user preferences. Next, a CAD model is generated automatically according to the user's scan-driven data. The model is based on a functional skeleton and a skin to customize appearance. This approach allows us to increase social acceptance while preserving a reasonable level of hand functionalities. In addition, according to the user preferences an interface is chosen. The available options range from simple bodypowered hands to more advanced EMG control and foot control interfaces, sensory feedback and more. This step ends with files entirely ready for 3D printing, a circuit board according to the chosen user interface and a bill of materials based on standard off-the-shelf parts. The last and final step is assembly. This step is the only manual one in the pipeline but is shortened significantly by optimizing the previous steps. The entire pipeline is illustrated in Fig. 1. The proposed pipeline and final outcome will potentially help to decrease cost, fitting-time, rejection rate and more. The entire research and final design are uploaded and shared online for anyone in the world to use and modify.

\section{SYSTEM ARCHITECTURE}

The purpose and implementation of each of the blocks in Fig. 1 are explained in detail in the following sections. Since we aim to create a low-cost design pipeline, the electronic design contains only off-the-shelf components. Moreover, the mechanical design was created such that the model can be manufactured by a standard 3D printer using standard printing materials such as ABS or PLA, and the assembly of the hand components would be simple to accomplish with basic tools. In addition, the user interface design was created for quick and simple adjustments according to the users' needs and desires and does not require a long training program to understand.

\section{A. Scan-Driven User Measurements}

One of the key aspects of our system is the personalization of the hand. Two aspects of the hand geometric structure are customized, the socket and the kinematic model. Currently the fitting of the socket is usually done manually by professionals thus resulting in long and costly procedures [11]. In our design we intend to use a lowcost 3D scanner and implement the resulting surface in our CAD model. While commercial 3D scanners are increasingly being used in the industry alongside the rise of 3D printers, these products are still expensive and unsuitable for our system. We offer a low-cost scanner design based on an Intel RealSense (Intel, Santa Clara, CA, USA) depth camera, shown in Fig. 2. The scanner is based on a single camera, and is almost entirely made from 3D printed parts, combining 3D printed thrust bearings, a single stepper motor, and four micro switches. The control is implemented with an Arduino board (Arduino, Italy). The production cost of this design is estimated at $\sim 40 \$$ on top of the RealSense camera. The camera rotates around the stump in a known trajectory and captures data in specific locations. The different point clouds are transformed into a single coordinate system to create one

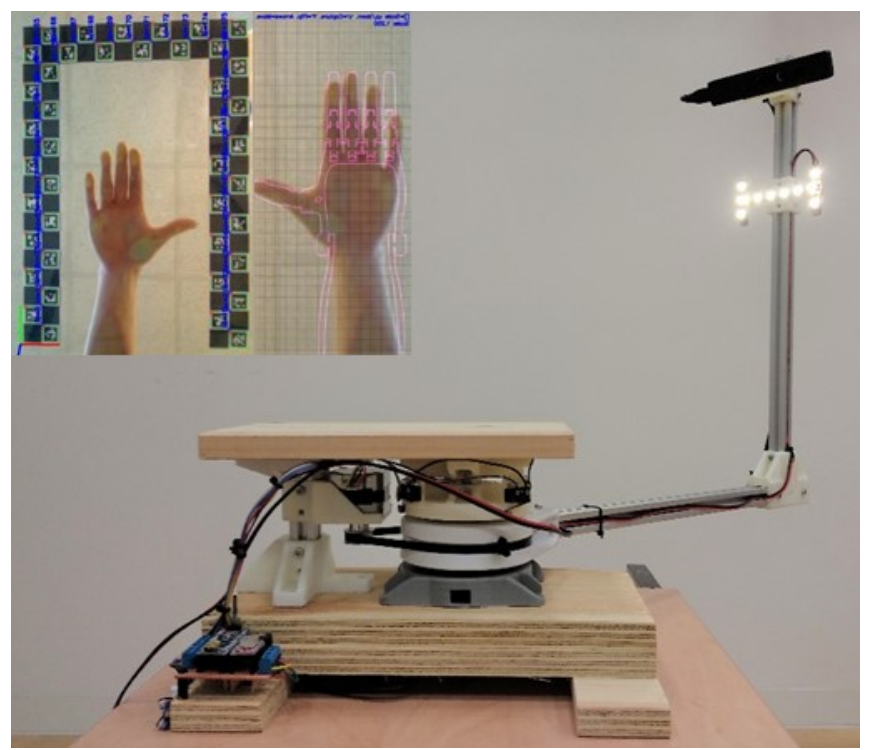

Figure 2. The low-cost 3D scanner based on a rotating RealSense camera. On the top left, the 2D scanner resulting raw image with the calibration markers, and the scaled image with a simple model fitted to the user's hand. 
point cloud. Once the point cloud is collected, a piece-wise parametric surface is fitted to the data. The current surfaces we use are Bezier surfaces, which are widely used in different applications and are suitable for our purpose for two main reasons. (i) The mathematical calculation is simple, meaning that the model can be generated in real-time and; (ii) The surface lies within the convex hull of the control points i.e. the entire scan is contained in the resulting surface. Such a surface can be integrated into our CAD model to generate the customized socket. The customized socket incorporates the needed sensors for the control interface and the sensory substitution actuators, all of which are discussed in subsequent sections. The suggested pipeline reduces the need of trained professionals thus significantly reducing cost.

In addition to the socket and unlike most existing designs, the terminal part of the prosthesis (the hand itself) is also customized according to the user's anatomical data. This is done using a regular camera mounted under a transparent plate. Printed markers are placed on the perimeter of the plate; the software locates these markers, fixes any distortions and scales the image to size. The interface and output are shown in Fig. 2. Using this system, our kinematic model is fitted to the user's unaffected hand. This data in-turn is passed to the CAD model to create a perfectly fitted hand design, as discussed in the following section.

\section{B. Prosthetic Hand Design}

The solution presented here is an electronic hand that can perform a variety of user-defined hand configurations and grips by actively controlling all the fingers independently with different levels of grip forces using micro DC motors. In general, the user's input is converted into the desired action by actuating specific motors and measuring the current drawn by each motor. According to current control, an adjustable grip is achieved based on the resistance that each motor undergoes. In parallel, a feedback signal is transmitted to the user that resembles the grip status and/or the object temperature. The desired action is completed as a result of the control system and/or the user's input. Hence, we essentially have two closed feedback loops as shown in Fig. 3. The first loop is between the controller and the actuators using the drawn current as the control signal and the second loop is between the user and the prosthetic hand using the feedback signal as the control signal.

\section{1) Mechanical Design}

As one would anticipate, the mechanical design is a significant part of our system. Our design is a bioinspired under-actuated hand and can be divided into two parts, a skeleton- kinematic structure and skin- for appearance. By splitting our design, we can optimize both functionality and appearance thus potentially reducing rejection rates. The entire CAD model is controlled via equations linked to the scan-driven user data mentioned in previous sections, through a graphical user interface (GUI) in MATLAB (MathWorks, Natick, MA, USA) as shown in Fig. 4A. The design was created in SolidWorks (Dassault Systèmes, Paris, France).

The skeleton shown in Fig. 4B determines our kinematic model and is based on commonly used hand models e.g. [14] the model has $20 \mathrm{DOF}$, three in the thumb, four in the other fingers (flexion-extension and abduction-adduction) and one in the wrist (pronation-supination). However, the hand is actuated using only six motors driving a tendon system, hence the design is highly under-actuated. This means that the finger closing profile is under-defined and is determined by the force equilibrium, thus allowing the hand to conform to various object shapes while maintaining a relatively small amount of active DOF [15]. This type of actuation is well suited to the type of control we have chosen and is described later in the text. To maximize grip success, the joints are pretensioned in a way that each finger closes gradually such that the proximal phalanx closes first, followed by the middle phalanx and lastly the distal phalanx.

As mentioned, social acceptance is one of the major rejection reasons. While younger users are in many cases interested in a robotic look, others prefer a human-like hand. The separation of the prosthetic into a skeleton and a skin allows us to satisfy both ends of the spectrum without the need for a kinematic redesign. Up till now, we have been working with two skins, a human-like skin and an "Ironman" skin, both are shown in Fig 1. We aim to simplify the process of incorporating a new skin into the design thus allowing any user to create or find a skin suitable for him\ her. Furthermore, using a 3D scanner such as the one offered, we can potentially mirror the scan of the user's unaffected hand and use it to create a truly personalized skin.

Once the kinematic model and skin are fitted to the user the output of our mechanical design is two CAD assembly files. The first is a functional assembly used to display all the parts connected, check for interferences and do a general evaluation. The second assembly is a print-ready assembly in which all the parts are laid out on a surface according to the printer bed size, in their ideal configuration for print. The orientations are optimized mainly to maximize strength but also to minimize support volume. This assembly saves valuable time when preparing the files for print.

\section{2) Electrical Design}

The electronic design was developed as a combination of

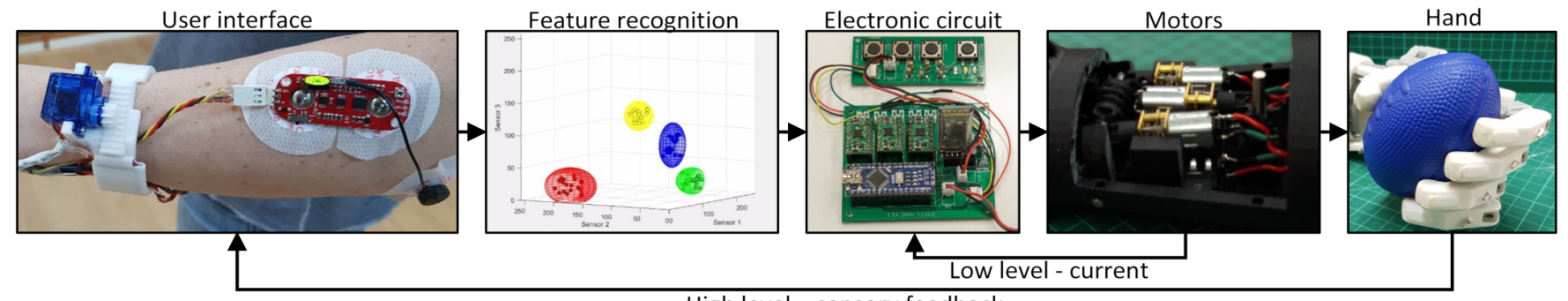

High level - sensory feedback

Figure 3. A block diagram of the control system consists of a low-level control solely on the controller and high-level control through the user interface. 


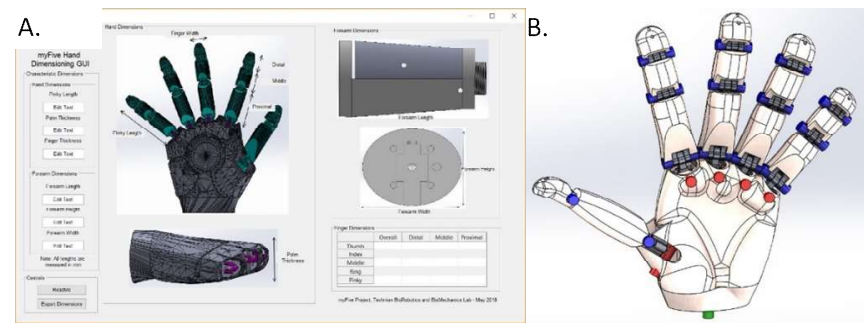

Figure 4. CAD Model. A. The same kinematic model with an Ironman skin in the GUI that controls the dimensions to create a personalized model. B. The human-like skin, the red blue and green cylinders represent the axes of rotation (are not part of the model).

modules to achieve maximum personalization of the device. The Printed Circuit Boards (PCB), shown in Fig. 3, were designed in EAGLE (Autodesk, San Rafael, CA, USA) and are published as open source designs. The main device circuit is the actuation control system and consists of the following: (i) Arduino Nano microcontroller was selected due to its low price, small size, analog inputs, low battery consumptions, and easy-to-use programming method. However, its low memory and small computational capacities require the development of efficient and module-like algorithms. (ii) Six low-powered micro DC motors. These tiny DC motors were chosen due to their size and weight. In addition, since the required voltage is low, a small and lightweight power supply can meet the system requirements. (iii) Three breakout boards (Pololu corporation, Las Vegas, NV, USA) with DRV8833 drivers (Texas Instruments, Dallas, TX, USA) were selected to drive and control the motor. This chip can drive two motors for both directions while measuring the current drawn by each motor using the overcurrent protection circuit of the chip. This breakout board was chosen due to its small size and versatility of the cut-off current. (iv) Power supply unit with one rechargeable and protected Lithium-Ion battery and a step-up circuit. The battery was selected to be able to supply at least 10 hours of average consumption. The connection between the user control circuit and the motor control system circuit is implemented as wireless communication via Bluetooth or wired communication through a multiplexer to enlarge the number of inputs to the microcontroller.

\section{3) Low level Control-Adaptive Grip}

The main functions of the modules are to (i) independently activate each motor according to the defined task sent by the user control module, (ii) apply an adaptive grip, and (iii) monitor the user's inputs status for force grip adjustment and user's safety. Once the user desires are captured in one of the possible user control systems, a hand motion is initiated. From this point, data on the grip status is gathered at the microcontroller and an adaptive grip is applied without the need for user inputs. The current control method which produces the desired adaptive grip is based on the current drawn by the motors when resistance is applied i.e. the prosthetic touches the object. The method uses three parameters - the absolute current value, slope and action time. These three parameters combined reduce the errors caused by the natural high starting current and the noise caused by the mechanical friction and leakage current. The implemented adaptive control mechanism allows automatic grip adaptation to objects of various size and shape, with different torque levels. This method of operation is similar to the haptic perception in a healthy hand where mechanoreceptors measure pressure and skin deformations as a result of grasping an object.

\section{User Interfaces}

One of the feedback received from prospective users and according to Cordella et al. [1] is about the reliability of the device. Since our hands are engaged with our environment most of the day, it is important that the electronics of the prosthetic hand and the methods to control it have high reliability over time. In addition, there is a need to control the prosthetic's motion while drawing as little visual attention as possible. Other features suggested include: controlling the grasping force, controlling each finger individually and adding more wrist motion before and during grasping an object [1]. Although all these requirements are important, a clear trade-off can be noticed. Increasing the number of usercontrolled functionalities and DOF may also increase training time, the cognitive burden and reduce the reliability and the classification accuracy of the user's motions. According to the above factors and more, we have developed several user and feedback interfaces.

\section{1) User Control}

The functionalities that can be controlled by the user are whether to move a specific finger and to which direction (i.e. opening or closing); the force level applied during grasping; and whether to rotate the wrist and to which angle. The core concept is to reduce the number of actions the user needs to perform and remember in order to accomplish the desired hand movement. Hence the prosthetic hand functionalities are designed as a tree-like structure.

One of the user control methods we developed is based on using the stump's muscle activation signal. The acquired signal can be an EMG signal or a force myography signal. As mentioned, the selected microcontroller has limited memory capacity and computational capabilities, hence a minimalistic way of analyzing the EMG signal was developed. The chosen MyoWare EMG sensors (Advancer Technologies, Raleigh, NC, USA) shown in Fig. 3, have a built-in signal processing hardware that outputs an amplified, rectified, and integrated signal. To capture and recognize the user muscle pattern, a calibration stage needs to be performed. During this stage the user can apply repetitive muscle movement that would be associated with one of the pre-defined hand movements. The classification method is based on the local peak value at a specific time window of each sensor. Hence, during the usage with the hand only specific values are classified as specific hand movement and the rest are considered as outliers. The main advantage of this method is that each user can define his own control inputs according to his muscle capabilities and skills. Moreover, the calibration stage can be applied at any time to overcome the known drawbacks of EMG sensors such as sensitivity to the adhesion quality of the skin, sweat, muscle fatigue and other environmental factors [19]; that might have a major effect on the classification accuracy of the user's desires.

However, controlling the prosthetic hand using the stump's muscles is not suitable for all amputation cases. E.g. amputees who suffer from phantom pain when using the residual limb [16] or people who suffer from permanent loss of muscular activity due to lack of physical activity and 
hence do not have a sufficient and reliable EMG signal, and muscle activation [12], [17]. Therefore, additional user control was developed to overcome this gap. It is imperative to develop a user control that will interfere as little as possible during daily activities. We have developed a very simple foot control which utilizes the change in the ankle diameter during dorsiflexion and plantar flexion. Using this method, we can robustly detect when the user is tapping with his foot on the floor or in the air and thus control opening and closing motions of the prosthetic.

In parallel, we are currently developing other methods to enrich the number of inputs from the user's feet that can be perceived and distinguished. This under-development work is focused on a gesture recognition of the foot, based on motion sensors (IMUs), pressure sensors and EMG sensors attached to the shoe, sole and to the ankle respectively.

\section{2) Sensory Feedback}

While much research is done on the subject, sensory feedback remains a challenge even in high-end prosthetic hands [18]. The issue of sensory feedback in low-cost designs is rarely discussed even though it is highly significant for proper function and device adoption [1]. The addition of sensory feedback has the potential to increase device functionality, mainly grasping capability. Also, current prosthetic users use the device as a tool, but sensory feedback might increase body ownership [19].

The main sensory modalities that are of interest are force feedback (which could indicate object slippage); texture and temperature. In our design we will focus on force feedback. To add the missing sensory components, our design includes a mechatronic system that samples force and temperature sensors embedded in the fingertips. The force sensors used, are force sensing resistors (FSR), FSR 400 short (Interlink Electronics, CA, USA). These sensors are flexible, durable, lightweight and inexpensive. The system provides the user with feedback using the feedback band shown in Fig. 5. The two motors apply force on the residual limb as a function of the force applied by the fingers and the location of the grasped object. In addition, the system provides vibrotactile feedback when it senses object slippage. Lastly, thermistors

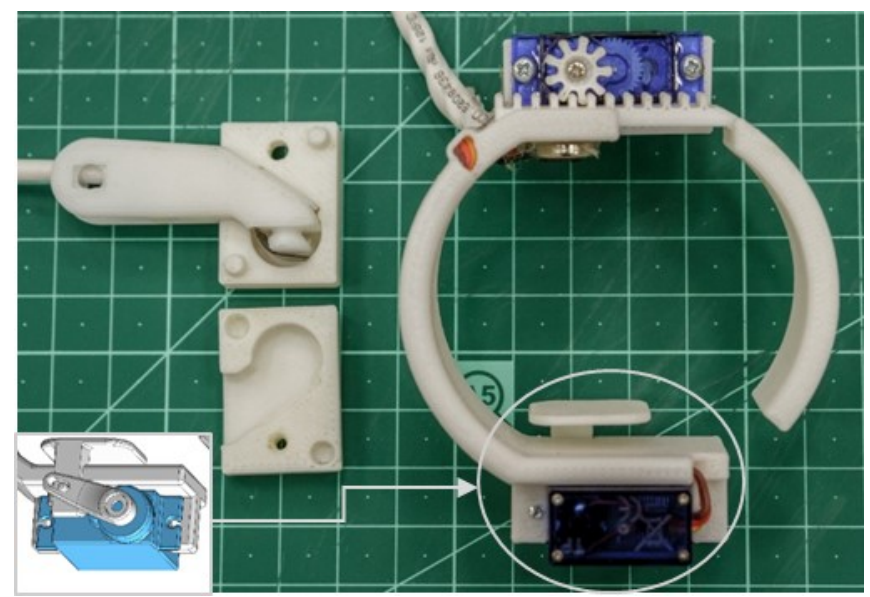

Figure 5. The force sensor embedded in the fingertip using moulds and the sensory substitution including- two servo motors to apply forces on the stump and a vibration motor to indicate slippage. The rear view of one of the servo motors can be seen at the bottom left corner. are connected to a buzzer and provide auditory feedback when the surrounding temperature could damage the prosthesis.

\section{PRELIMINARY RESUltS}

\section{A. Mechanical Hand Design}

The device was tested in common ADLs including, holding a glass, bottle, and a can; pouring liquid and particles from one container to another; gripping a ball, notebook, phone, and keys; performing gestures and writing. From all the above ADLs, only writing was not simple to accomplish, and the task could be achieved only when using a large marker. Moreover, each actuated element of the model was tested over $50 \mathrm{~K}$ cycles to ensure reliability and durability of the design.

\section{B. Adaptive Grip}

Our results also demonstrated that the hand has an adaptive grip. As a result, the prosthetic capabilities include opening, closing, grasping, and lifting objects with different shapes and weight with variable grip force. Several evaluations were performed to ensure the correlation between the current control and the grasping behavior process. Fig. 6 demonstrates two examples of different objects grasping stiff object and soft object. At first, each movement starts with a high current drawn by the motor to initiate a motion. Then, a low current is measured for each motor until the grasping has started. At this point, different current behavior can be detected. When the fingers are holding a stiff object a steady and relatively moderate slope can be detected, and hence a different torque level can be applied to induce different grip force pattern. Also, it can be observed that each motor stops at a different time, thus allowing the adaptive grip technique. In this example, a tennis ball represented the stiff object, and indeed a correlation is visible where first the index finger completed the gripping task, followed by the middle, ring and lastly the pinky finger, similar to a power sphere grasp made by a human hand. When holding a soft object, the resistance sensed by the motor changes along the grasping process. At the beginning a high current is drawn by the motor due to the impact when the finger encounters the object, next, the elasticity of the soft object reduces the resistance between the object and the finger and the current drawn by the motor decreases until the object is compressed enough to increase the current again.

\section{Feedback from Prospective Users}

Although a formal feedback collection has not yet been accumulated in the framework of this paper, initial feedback from prospective users and feedback from healthy subjects was collected. One of the main benefits raised by the prospective users after examining the hand is the low overall weight ( $\sim 300 \mathrm{gr})$ of the prosthetic hand in comparison to current high-end prosthetic hands. In addition, although, there are more hand configurations than needed, users were appreciative of several gestures configurations that are meant to be more for communication than for grasping and holding. This type of configuration cannot be found in most of the commercial prosthetic hands and was inspired by the natural human hand's use in communication and entertainment and not only for functional tasks. 
Holding a Stiff Object : Closing all four Fir

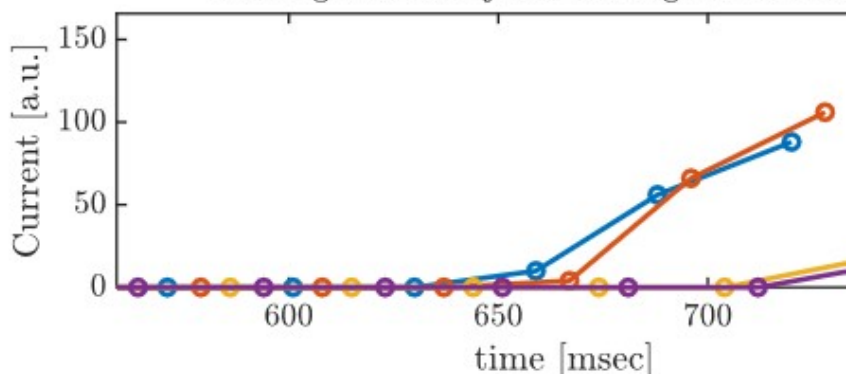

Holding a Soft Object : Closing all four Fir

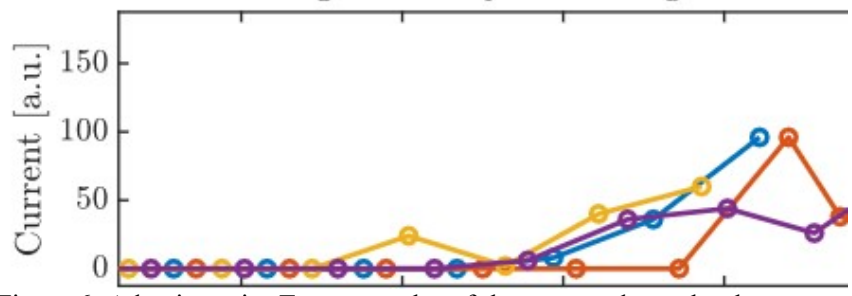

Figure 6. Adaptive grip. Two examples of the current drawn by the motors during grasping a stiff object, and a soft object, at the end of the fingers motion.

\section{CONCLUSION AND FUTURE WORK}

Most existing prosthetic hands have a very high price tag; this means that they do not offer a feasible solution to large portions of the population and especially to children due to constant growth. The price is determined in many cases by the manual fitting procedures involved, and by the use of high-end hardware even when it is not mandatory. Rejection rates remain high even when financial resources do not present an issue. In this paper, we present a fully automatic fitting pipeline concept for a personalized, lowcost 3D printed prosthetic hand. The fitting concept is based on the extraction of the user geometric data i.e. a 3D scan of the stump and kinematic structure of the non-affected hand. The 3D design is adjusted accordingly. In addition, the appearance of the hand can be easily modified according to user preferences. The hardware was chosen as minimalistic as possible and providing only essential features, thus resulting in a simple and robust design. By designing a modular software and hardware we allow the user to choose the most suitable interface for him $\backslash$ her. Currently, we offer an EMG interface and a foot-based interface. Lastly, the output of the automated pipeline is an easy to manufacture design with a minimal need for manual adjustments. The above-mentioned advantages could enable intensive use by users who reject other prosthetic hands and were looking for a better solution to their situation, we believe this concept has the potential to provide a much-needed leap in the field.

\section{ACKNOWLEDGMENT}

We would like to thank Oded Katzman and the myFive team for their help with the mechanical design, Guy Medan for his help with the 2D scanner, and Haifa3D organization for their ongoing support.

\section{REFERENCES}

[1] F. Cordella et al., "Literature review on needs of upper limb prosthesis users," Front. Neurosci., vol. 10, no. MAY, pp. 1-14, 2016.

[2] E. Strait, G. McGimpsey, and T. C. T. Bradford, "Limb prosthetics services and devices," Bioeng. Inst. Cent. Neuroprosthetics Worcester Polytech. Inst., no. January, pp. 1-35, 2008.

[3] M. Meurs, C. G. B. Maathuis, C. Lucas, M. Hadders-Algra, and C. K. van der Sluis, "Prescription of the first prosthesis and later use in children with congenital unilateral upper limb deficiency: A systematic review," Prosthet. Orthot. Int., vol. 30, no. 2, pp. 165-173, 2006.

[4] K. Huizing, H. Reinders-Messelink, C. Maathuis, M. Hadders-Algra, and C. K. Van Der Sluis, "Age at first prosthetic fitting and later functional outcome in children and young adults with unilateral congenital below-elbow deficiency: A cross-sectional study," Prosthet. Orthot. Int., vol. 34, no. 2, pp. 166-174, 2010.

[5] D. Katsavelis, A. Carson, J. Peck, R. Srivastava, and J. M. Zuniga, "An Open Source 3D-Printed Transitional Hand Prosthesis for Children," J. Prosthetics Orthot., vol. 28, no. 3, pp. 103-108, 2016.

[6] C. Schubert, M. C. Van Langeveld, and L. A. Donoso, "Innovations in 3D printing: A 3D overview from optics to organs," $B r . J$. Ophthalmol., vol. 98, no. 2, pp. 159-161, 2014.

[7] J. ten Kate, G. Smit, and P. Breedveld, "3D-printed upper limb prostheses: a review," Disabil. Rehabil. Assist. Technol., vol. 12, no. 3, pp. 300-314, 2017.

[8] J. L. Parry-Hill and D. L. Ashbrook, "Challenges and Opportunities in DFO-AT: A Study of e-NABLE," 2016.

[9] L. Dichen, L. Gang, H. Sanhu, S. Singare, L. Yaxiong, and L. Bingheng, "Fabrication of customised maxillo-facial prosthesis using computer-aided design and rapid prototyping techniques," Rapid Prototyp. J., vol. 12, no. 4, pp. 206-213, 2006.

[10] A. Cappello, R. Gori, M. Viceconti, D. Testi, A. De Lollis, and C. Zannoni, "HIDE: a new hybrid environment for the design of custommade hip prosthesis," Comput. Methods Programs Biomed., vol. 64, no. 2, pp. 137-144, 2002

[11] C. Rizzi, G. Colombo, and G. Facoetti, "A digital patient for computer-aided prosthesis design," Interface Focus, 2013.

[12] M. Atzori and H. Müller, "Control Capabilities of Myoelectric Robotic Prostheses by Hand Amputees: A Scientific Research and Market Overview," Front. Syst. Neurosci., vol. 9, no. November, pp. $1-7,2015$.

[13] L. J. Resnik et al., "EMG pattern recognition compared to foot control of the DEKA Arm," PLoS One, vol. 13, no. 10, pp. 1-16, 2018.

[14] F. Chen Chen, S. Appendino, A. Battezzato, A. Favetto, M. Mousavi, and F. Pescarmona, "Constraint study for a hand exoskeleton: Human hand kinematics and dynamics," J. Robot., vol. 2013, 2013.

[15] J. T. Belter, J. L. Segil, A. M. Dollar, and R. F. Weir, "Mechanical design and performance specifications of anthropomorphic prosthetic hands: A review," J. Rehabil. Res. Dev., vol. 50, no. 5, p. 599, 2013.

[16] S. L. Carey, D. J. Lura, and M. J. Highsmith, "Differences in myoelectric and body-powered upper-limb prostheses: Systematic literature review," J. Rehabil. Res. Dev., vol. 52, no. 3, pp. 247-262, 2015.

[17] Z. Xiao, B. Pousett, L.-K. Merhi, C. Menon, E. Cho, and R. Chen, "Force Myography to Control Robotic Upper Extremity Prostheses: A Feasibility Study," Front. Bioeng. Biotechnol., vol. 4, no. March, pp. $1-12,2016$.

[18] J. S. Schofield, K. R. Evans, J. P. Carey, and J. S. Hebert, "Applications of sensory feedback in motorized upper extremity prosthesis: A review," Expert Rev. Med. Devices, vol. 11, no. 5, pp. 499-511, 2014.

[19] U. Wijk and I. Carlsson, "Forearm amputees' views of prosthesis use and sensory feedback," J. Hand Ther., vol. 28, no. 3, pp. 269-278, 2015. 